\title{
The Socio-technical Constitution of Resilience: Structures, Practices, and Epistemologies
}

\author{
Kurniawan Adi Saputro
}

Graduae School of Indonesia Institute of the Arts Yogyakarta

On 21-22 June 2016, School of Humanities and Social Sciences, Nanyang Technological University and Future Resilient Systems, Singapore-ETH Centre held a conference on socio-technical constitution of resilience. Twenty eight scholars from as various disciplines as humanities to civil engineering convened and discussed the subject from various angles. At the centre of attention was the concept of resilience, which inherited its meaning from socio-ecological system research, referring to its capacity to absorb disturbance and maintain its integrity. However, the concept was recast for a more specific context, namely sociotechnical context, characterised by intentions of both human and technological agents. In such specific context, resilience comprised of three core aspects: its informational relations, its sociomaterial structures, and its anticipatory practices (Amir and Kant, 2017). Informational relations referred to "how information is organised and managed to support continued operation of sociotechnical system" (ibid.), while sociomaterial structures referred to "mutual entanglement of human organisation and material structures" (ibid.). Finally, anticipatory practices referred to "construction of regular activities aimed at anticipating possibilities of what would occur in the future" (ibid.).

These three aspects of sociotechnical resilience were greatly expanded and enriched by case studies, ranging from water scarcity in Panama, makeshift dwelling in Hyderabad, scientific discourse of Sidoarjo mudflow, cross-media audiences of Mt. Merapi eruption, and many others. In addition to case studies, several researchers problematised resilience by politicising and historicising the verb, by complementing systemic perspective with situated perspectives, by diversifying its epistemologies, and by casting it as an active participation by citizens. Case studies and concept problematisations were strategies to approach a problem as hard to resolve as resilience since it encompasses different, often contradictory, dimensions. For example, technical systems require rigid and exact protocols and standardisation, whereas social systems are inherently and 
promote flexible and negotiable arrangements. This is only one of many problems that refuse easy solutions, maybe an inexistent path to choose. Accordingly, participants were eager to localise the problems in different contexts, approached it carefully and holistically, and attempted to locate the concept in a certain historical trajectory to not lose sight of its limitations and biases. The following parts will report the discussions chronologically as they happened in the two days of conference.

Ashley Carse discussed the case of water scarcity in Panama as an "infrastructural event" since it was not something that was inevitable but produced by the country's policy choices to prioritise their economic development relying on their ship canals. Hence, in the face of El-nino related droughts, they wanted households to limit their consumption of water while continuing to increase the capacity, and water supply, of its canal. Stephen Healy problematised the current conception of resilience as a property in "systems ecology" that can universally be found despite differences in cultures and histories. He proposed complementing the universalising tendencies of Western thought with more particularistic and situated perspective. In his words an assemblages "gather together not only the technical elements specific to particular places or processes but also the social and moral meanings, and agential forces that people bring to bear along with these technical elements to produce circumstances in which events occur" (p. 42). The two paper had shown that resilience, and vulnerability as its flip side, were as complex as society's historical developments, people's meaning-making, and particular usage of technical instruments.

Makoto Takahashi and Masaharu Kitamura studied the incident of Fukushima Daiichi nuclear power plant failure that resulted in its meltdown and evacuation of approximately 100,000 people. They found that the incident could have been worse it it was not due to the current good practice conducted by the personnels. Shin-etsu Sugawara and Kohta Joraku examined how the Fukushima highlighted the complicated nature of its real-time simulation technology, called SPPEDI, which did not perform sufficiently, created controversies among governmental bodies. The technology of warning was also examined by Jennifer Henderson in her study of Weather Forecast Offices in the US, which found that the meteorologists shifted their practice from making better and accurate predictions to interpreting and communicating the findings to different government bodies and the publics. The shift to informing the "customers" and making impacts created an ethics of resilience, in which they orientate their scientific knowledge and institutional practices toward making the wider public more resilient, rather than merely recording information and making predictions without clear guidance as to how to interpret and use the predictions.

Diganta Das observed how slum-dwellers in high-tech hub city of Hyderabad showed resiliency despite losing their lands and primary source of income. He focused on spatial dimension of people's resilient practices, in claiming and governing their own private and communal spaces. Scott Gabriel Knowles and 
Jose Torero reframed the problems of universal versus particular approaches to resilience to a problem of command-and-control versus historico-political realities of vulnerability. By focusing on how political struggles unfolded between managements, engineers, and public health overseers, the authors revealed that the current focus on resiliency concerned more about winning society's agenda, rather than ensuring that the inventories of weaknesses and vulnerabilities had been dealt with. Vivek Kant and Justyna Tasic proposed to blend top-down with bottom-up approach by starting with the activities in the bottom, then moving to discovering relations. They made use of the concept of holons to identify parts of the system that simultaneously functioned as a whole, namely purpose, act, agent, scene, and agency. Socio-technical systems as meshworks of holons provided insight into resilience as one of its systemic properties.

Small business provided insights into soft non-structural measures of resilience in the form of financial support for small and medium enterprise's business operations, as had been studied by Bingunath Ingirige and Gayan Wedawatta. Another form of "soft" measure to increase resilience post-factum was to create an authoritative report of accidents so that a nation could "reach consensus". Chihyung Jeon, Hyungsub Choi, and Sungeun Kim found three types of reports, namely legal, technical, and bureaucratic reports of accidents since 1990s in South Korea. These three types of reports served "narrow institutional needs", while neglecting social and structural narratives. Ryuma Shineha and Mikihito Tanaka drew conference's attention to gaps between national and local media focus on disaster. In Japan after the March 2011 Tohoku earthquake, local media did not have the power to draw national media's attention toward issues of their concern, which did not improve on their resilience. Anto Mohsin discussed the ambiguities of map as an instrument of power (to contain and manage affected population) and instrument to assert rights (by affected citizens themselves). His study of Lapindo mud in East Java show how murky the scientific claims of causality and government's attempt at mitigating the impacts, exemplified by the map that kept changing during the ongoing crisis.

Charlotte Cabasse problematised the underlying assumption of resilience as a move toward normalcy, which contradicted the changing, abnormal reality of Polynesian people affected by climate change. Megan Finn's study on public information infrastructure revealed that following events of disaster, state reasserted its authority to define what information infrastructure was and should be using the language of resilience, although the infrastructure itself was privately owned. In other words, resilience opened the private infrastructure of information for state's intervention.

The last two papers concerned seeing resilience as a distributed capacity of the society, enhanced by the unprecedented capacities of modern information and communication technologies. Kurniawan Adi Saputro offered a model to understand mediated disaster as a move from society's collective attention, mediated by media, toward collective action to help the affected people. The 
move was uncertain and faced with different challenges, yet could show great potential as was shown in the successful disaster relief efforts in the wake of Mt. Merapi eruption in Indonesia 2010. Katrina Petersen and Monika Buscher reported their project to design ICT for "spatially and culturally distributed disaster management" (p. 306). It went beyond security versus freedom discourse and moved toward identifying risks as they were realised in complex interactions based on "data, technology, and movement" over borders. The result of this conference will be published in anthology on sociotechnical resilience sometimes in the year of 2018. 